\title{
Smoothed particle hydrodynamics modeling of linear shaped charge with jet formation and penetration effects
}

\author{
D.L. Feng ${ }^{\text {a }}$ M.B. Liu ${ }^{\mathrm{a}, *}$, H.Q. Li ${ }^{\mathrm{b}, *}$, G.R. Liu ${ }^{\mathrm{c}}$ \\ a Institute of Mechanics, Chinese Academy of Sciences, Beijing 100190, China \\ ${ }^{\mathrm{b}}$ School of Information and Electronics, Beijing Institute of Technology, Beijing 100081, China \\ ${ }^{\mathrm{c}}$ Aerospace Systems, University of Cincinnati, Cincinnati, OH 45221-0070, United States
}

\section{A R T I C L E I N F O}

\section{Article history:}

Received 23 October 2012

Received in revised form 11 April 2013

Accepted 28 June 2013

Available online 5 July 2013

\section{Keywords:}

Shaped-charge jet

Smoothed particle hydrodynamics (SPH)

Penetration

Kernel gradient correction

\begin{abstract}
A B S T R A C T
Shaped charge, as a frequently used form of explosive charge for military and industrial applications, can produce powerful metal jet and lead to stronger penetration effects onto targets than normal charges. After the explosion of high explosive (HE) charge, the detonation produced explosive gas can exert tremendous pressure on surrounding metal case and liner with very large deformation and even quick phase-transition. In this paper, the entire process of HE detonation and explosion, explosion-driven metal deformation and jet formation as well as the penetrating effects is modeled using a smoothed particle hydrodynamics (SPH) method. SPH is a Lagrangian, meshfree particle method, and has been widely applied to different areas in engineering and science. A modified scheme for approximating kernel gradient (kernel gradient correction, or KGC) has been used in the SPH simulation to achieve better accuracy and stability. The modified SPH method is first validated with the simulation of a benchmark problem of a TNT slab detonation, which shows accurate pressure profiles. It is then applied to simulating two different computational models of shaped-charge jet with or without charge cases. It is found that for these two models there is no significant discrepancy for the length and velocity of the jet, while the shapes of the jet tip are different. The modified SPH method is also used to investigate the penetrating effects on a steel target plate induced by a linear shaped charge jet. The effectiveness of the SPH model is demonstrated by the good agreement of the computational results with experimental observations and the good energy conservation during the entire process.
\end{abstract}

() 2013 Elsevier Ltd. All rights reserved.

\section{Introduction}

A shaped charge is generally a high explosive charge with a hollow cavity (usually cone shape) at one end with a thin layer of liner, and a detonator at the opposite end (illustrated in Fig. 1) [1-3]. The gaseous products produced in the detonation process can exert extremely high pressure and high temperature on surrounding case and liner (usually a metal, such as copper or aluminum), causing very large deformation and even liquefaction. When detonation shock engulfs the lined cavity, the softened or liquefied liner moves towards the centerline or charge axis of symmetry, and forms a metal jet with large kinetic energy. With the evolution of the HE detonation process, the metal jet is gradually accelerated and elongated with increasing velocity until the HE detonation process completes. The focusing of the metal jet can create an intense localized force, which is capable of creating a deeper crater on a plate than that created by explosive charges without a hollow

\footnotetext{
* Corresponding authors. Tel.: +86 1082544024 .

E-mail addresses: fengdianlei@imech.ac.cn (D.L. Feng), liumoubin@imech.ac.cn (M.B. Liu), huiqili@bit.edu.cn (H.Q. Li), liugr@ucmail.uc.edu (G.R. Liu).
}

cavity, even though more explosive is available in the latter cases. Therefore shaped charges are commonly used in military for penetrating hardened tanks and targets, and in industry for cutting metals, rocks and mineral layers through using a specially designed charge devices. The shaped charge devices can usually be categorized into two classes, linear shaped charges and cylinder shaped charges. Fig. 2 shows an illustration of a typical linear shaped charge.

Investigations of shaped charges and the corresponding damaging effects date back to several decades ago. Though many researchers have conducted a large amount of work using theoretical analysis, numerical modeling, and laboratory or even field experiment, publically available literature is limited due to the restrictions from military establishments. Theoretical analyses on shaped charges usually start from simplified assumptions, and then are tested and corrected with experimental observations. For example, by assuming that the pressure of the explosive gas drops to zero as soon as the liner start to collapse and the liner material behaves like an incompressible inviscid fluid, Birkhoff et al. [2] provided a theoretical analysis of the shaped charge jet based on Bernoulli's equation. The validity of similar theoretical 


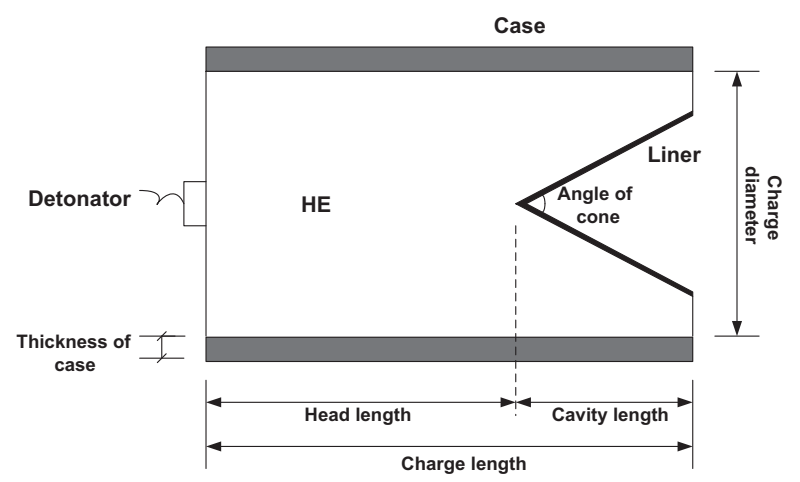

Fig. 1. An illustration of a typical shaped charge in two dimensional spaces.

analyses is experimentally verified later by Eichelberger [4]. In experiment, Green obtained the first X-ray photograph of a shaped charge jet in 1974 [5]. Raftenberg presented an experimental investigation of RHA (Rolled Homogeneous Armor) plate perforation by a shaped-charge jet [6]. Yu et al. also provided an experimental investigation of a shaped charge on the jet formation and its penetration effects onto rocks [7]. However, experimental works are generally expensive and sometimes certain physical phenomena related to shaped charges cannot be scaled in a practical experimental setup. Therefore, experimental works are usually combined with numerical simulations for detailed analyses. For example, through comparing numerical results and experimental observations, Wang et al. investigated the metal jet formation of a shaped charge with a copper liner [8]. Katayama et al. studied the penetration process of a shaped with an aluminum liner by an inhibited shaped charge launcher experimentally and numerically with commercial software, AUTODYN-2D [9].

With the advancement of the computer hardware and computational techniques, more and more researches on shaped charges are focused on numerical simulations. Computer simulation enables parametric studies to be carried out without having to resort to expensive firing trials, and is very useful in designing shaped charges or protection systems against shaped charges. Some wave propagation hydro-codes [10-16], which were originally developed to solve problems characterized by the presence of shock waves, localized materials response and impulsive loadings, have been tried to simulate shaped charges. Most of these applications are generally grid-based numerical methods such as the finite element methods (FEM) or finite difference methods (FDM). Some of them are associated with combined features such as Arbitrary Lagrange-Eulerian (ALE) coupling and Coupling Eulerian-Lagrangian (CEL) $[17,18]$. For example, Molinari simulated the shaped charge jet formation using finite element method [19]. Ayisit investigated the influences of geometric symmetry of the shaped charge device

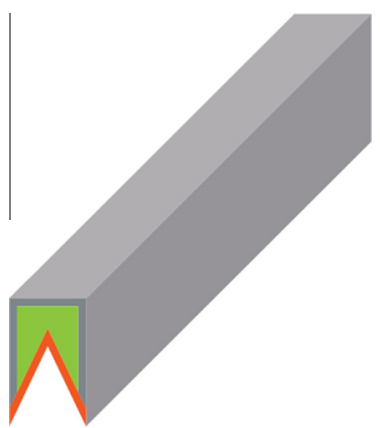

Fig. 2. An illustration of a typical linear shaped charge.
[20], while Karlsson also simulated the shaped charge jet formation [21]. In both work, a commercial software, AUTODYN, is used, while jet and target plate are computed within Euler and Lagrange frame separately. Though many successful achievements have been made for these methods in modeling shaped charges, some numerical difficulties still exist. These numerical difficulties generally arise from large deformations, large inhomogeneities, and moving interfaces, free or movable boundaries when simulating shaped charges including HE detonation and explosion, explosion-driven metal deformation and jet formation, target damaging and penetration.

Recently growing interests have been focused on the meshfree methods, which are expected to be superior to the traditional FDM and FEM, especially for applications with moving features such as free surfaces, evolutionary interfaces and large deformations. Among the meshfree methods, smoothed particle hydrodynamics (SPH) method [22-24] is unique in computational fluid and solid dynamics. As a comparatively new computational method, SPH combines the advantages of meshfree, Lagrangian and particle methods. First, in SPH, particles are used to represent the state of a system and these particles can freely move according to internal particle interactions and external forces. Therefore it can naturally obtain history of fluid/solid motion, and can easily track material interfaces, free surfaces and moving boundaries. The meshfree nature of SPH method remove the difficulties due to large deformations since SPH uses particles rather than mesh as a computational frame to approximate related governing equations. These features of SPH make it fairly attractive in modeling high explosive detonation and explosion, underwater explosion, and hydrodynamics with material strength such as impact and penetrations [25-31].

There are also some preliminary works of using SPH to model shaped charges. For example, Liu et al. first simulated the detonation and explosion process of two-dimensional shaped charges with different shapes of cavity [32] using SPH method. It is found that SPH can effectively model the explosive gas jet formation and dispersion. The work did not consider surrounding metal case and liner which present additional challenges in numerical simulation due to the existence of multi-material (explosive-metal) and multi-phase (solid-gas-liquid). Yang et al. also provided an $\mathrm{SPH}$ simulation of shaped charge jet formation in which both the explosive and metal liner are considered [33]. Existing works on SPH modeling of shaped charge is usually based on conventional SPH method, which is believed to have poor performances especially in modeling problems with highly disordered particles [25]. They lack quantitative and even qualitative comparisons with experimental results, and also lack validation and verification in energy conservation.

In this paper, we shall present a modified SPH model for simulating linear shaped charges with HE detonation and explosion, and hydrodynamics with material strength including explosion-driven metal deformation and jet formation as well the penetrating effects onto a target plate. As the size of a linear-shaped in longitudinal direction is much larger than those in other two directions, the linear shaped charge can be modeled as a plane strain problem in a two-dimensional space. Three numerical examples shall be provided to validate the effectiveness of the SPH model in simulating linear shaped charges.

\section{Smoothed particle hydrodynamics (SPH)}

\subsection{Basic concept of SPH}

SPH was originally invented to solve astrophysical problems in three dimensional open spaces as the collective movement of those 
particles is similar to the movement of a liquid or gas flow, and it can be modeled by the governing equations of the classical Newtonian hydrodynamics [22,23]. Due to its special features and advantages, it has been applied to various areas in engineering and sciences [24].

In SPH, particles are used to represent the state of a system and these particles can freely move according to internal particle interactions and external forces. Governing equations are also approximated within the frame of particles. The essentials of the SPH approximations are as follows. First, field variables and the corresponding derivatives are approximated as integral representation in continuous form using a kernel or smoothing function, and this step is referred to as kernel approximation. Secondly, the computational domain is discretized with a set of particles, and field variables and the corresponding derivatives are approximated as weighted summations over surrounding particles within the support domain of the smoothing function (illustrated in Fig. 3). As such, a field function and its derivative can then be written in the following forms

$$
\begin{aligned}
& \left\langle f\left(\boldsymbol{x}_{i}\right)\right\rangle=\sum_{j=1}^{N} \frac{m_{j}}{\rho_{j}} f\left(\boldsymbol{x}_{j}\right) W\left(\boldsymbol{x}_{i}-\boldsymbol{x}_{j}, h\right), \\
& \left\langle\nabla f\left(\boldsymbol{x}_{i}\right)\right\rangle=\sum_{j=1}^{N} \frac{m_{j}}{\rho_{j}} f\left(\boldsymbol{x}_{j}\right) \nabla_{i} W_{i j},
\end{aligned}
$$

where $\left\langle f\left(\boldsymbol{x}_{i}\right)\right\rangle$ is the approximated value of particle $i ; f\left(\boldsymbol{x}_{j}\right)$ is the value of $f(\boldsymbol{x})$ associated with particle $j ; \boldsymbol{x}_{i}$ and $\boldsymbol{x}_{j}$ are the positions of corresponding particles; $m$ and $\rho$ denote mass and density respectively; $h$ is the smooth length; $N$ is the number of the particles in the support domain; $W$ is the smoothing function representing a weighted contribution of particle $j$ to particle $i$. If representing the distance between particle $i$ and $j$ as $r_{i j}$, the smoothing function and its first order derivative can be expressed as follows:

$W_{i j}=W\left(\boldsymbol{x}_{i}-\boldsymbol{x}_{j}, h\right)=W\left(\left|\boldsymbol{x}_{i}-\boldsymbol{x}_{j}\right|, h\right)$,

$\nabla_{i} W_{i j}=\frac{\boldsymbol{x}_{i}-\boldsymbol{x}_{j}}{r_{i j}} \frac{\partial W_{i j}}{\partial r_{i j}}=\frac{\boldsymbol{x}_{i j}}{r_{i j}} \frac{\partial W_{i j}}{\partial r_{i j}}$.

where $r_{i j}$ is the distance between particle $i$ and $j$. The smoothing function is sometimes referred to as kernel or kernel function, and it should satisfy some basic requirements, such as normalization condition, compact supportness, and Delta function behavior. These conditions are needed to ensure the convergence and reproducibility of function approximation. Detailed discussions on the

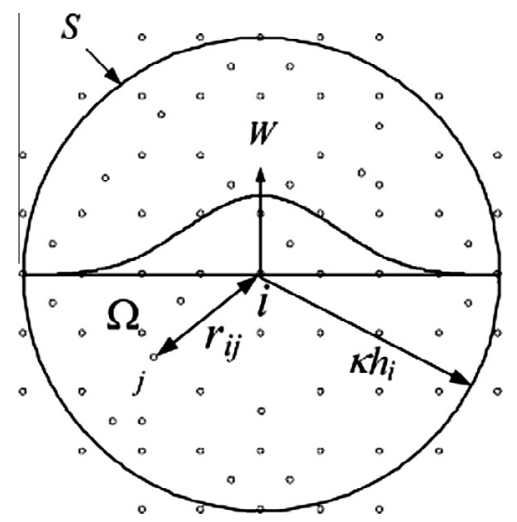

Fig. 3. An illustration of SPH approximations with a smoothing function $W$. The support domain of the smoothing function is a circle with a radius of $\kappa h$, in which $h$ is the smoothing length, and $\kappa$ is a scalar factor. smoothing function, its basic requirements and constructing conditions can found in [24]. In this paper, the following frequently used Gaussian kernel is employed

$W(S, h)=\alpha_{d} \mathrm{e}^{-S^{2}}$,

where $S=\left|\boldsymbol{x}-\boldsymbol{x}^{\prime}\right| / h, \alpha_{d}$ is a dimension-dependent constant related to the smoothing length. In one, two or three-dimensional space, $\alpha_{d}=\frac{1}{\pi^{\frac{1}{2}} h}, \frac{1}{\pi h^{2}}$ or $\frac{1}{\pi^{\frac{3}{2}} h^{3}}$ respectively. The scalar factor $\kappa$, used in this Gaussian kernel is 3 .

From Eqs. (1) and (2), it is seen that in SPH, numerical approximations for a field functions and its derivatives are based on discrete particles rather than on a mesh or grid system in the traditional FDM and FEM. There is no constrained connectivity between SPH particles, and therefore it is a truly meshfree method. This truly meshfree nature is especially attractive for problems with large deformations and moving features which usually challenge conventional FEM and FDM.

\subsection{SPH equations of motion}

In simulating linear shaped charges, the entire process include HE detonation and explosion, and hydrodynamics with material strength including explosion-driven metal deformation and jet formation as well the penetrating effects onto a target plate. For hydrodynamics of fluids and solids with material strength, the following governing equations of continuum mechanics apply

$$
\left\{\begin{array}{l}
\frac{D \rho}{D t}=-\rho \frac{\partial \boldsymbol{v}^{\beta}}{\partial \boldsymbol{x}^{\beta}} \\
\frac{D \boldsymbol{v}^{\alpha}}{D t}=\frac{1}{\rho} \frac{\partial \sigma^{\alpha \beta}}{\partial \boldsymbol{x}^{\beta}} \\
\frac{D e}{D t}=\frac{\sigma^{\alpha \beta}}{\rho} \frac{\partial \boldsymbol{v}^{\alpha}}{\partial \boldsymbol{x}^{\beta}} \\
\frac{D \boldsymbol{x}^{\alpha}}{D t}=\boldsymbol{v}^{\alpha}
\end{array},\right.
$$

where the scalar density $\rho$, and internal energy $e$, the velocity component $\boldsymbol{v}^{\alpha}$, and the total stress tensor $\sigma^{\alpha \beta}$ are the dependent variables. The spatial coordinates $\boldsymbol{x}^{\alpha}$ and time $t$ are the independent variables. The summation in Eq. (6) is taken over repeated indices, while the total time derivatives are taken in the moving Lagrangian frame. The total stress tensor $\sigma^{\alpha \beta}$ in Eq. (6) is made up of two parts, one part of isotropic pressure $p$ and the other part of shear stress $S^{\alpha \beta}$. The hydrodynamic pressure is computed from an equation of state (EOS). For explosive gas, as the isotropic pressure is much larger than components of viscous shear stress, the viscous shear stress can be neglected. For solid materials, the shear stress can be computed from the constitutive equations of corresponding materials. Therefore using above-mentioned SPH approximations, the following SPH equations of motion can be obtained

$$
\left\{\begin{array}{l}
\frac{d \rho_{i}}{d t}=\rho_{i} \sum_{j=1}^{N} \frac{m_{j}}{\rho_{i}}\left(\boldsymbol{v}_{i}^{\beta}-\boldsymbol{v}_{j}^{\beta}\right) \frac{\partial W_{i j}}{\partial x_{i}^{\beta}} \\
\frac{d \boldsymbol{v}_{i}^{\alpha}}{d t}=-\sum_{j=1}^{N} m_{j}\left(\frac{\sigma_{i}^{\alpha \beta}}{\rho_{i}^{2}}+\frac{\sigma_{j}^{\alpha \beta}}{\rho_{j}^{2}}+\Pi_{i j}\right) \frac{\partial W_{i j}}{\partial x_{i}^{\beta}} \\
\frac{d e_{i}}{d t}=\frac{1}{2} \sum_{j=1}^{N} m_{j}\left(\frac{P_{i}}{\rho_{i}^{2}}+\frac{P_{j}}{\rho_{j}^{2}}+\Pi_{i j}\right)\left(\boldsymbol{v}_{i}^{\beta}-\boldsymbol{v}_{j}^{\beta}\right) \frac{\partial W_{i j}}{\partial x_{i}^{\beta}}+\frac{1}{\rho_{i}} S_{i}^{\alpha \beta} \varepsilon_{i}^{\alpha \beta}+H_{i} \\
\frac{d \boldsymbol{x}_{i}^{\alpha}}{d t}=\boldsymbol{v}_{i}^{\alpha}
\end{array},\right.
$$

where $\varepsilon^{\alpha \beta}$ is the strain rate tensor, $\Pi$ and $H$ stand for the component of the deviator stress tensor, the artificial viscosity and the artificial heat separately [24].

\subsection{Artificial viscosity}

The artificial viscosity is used in SPH method to stabilize the numerical scheme, prevent particle penetration and capture shock 
waves. In this paper, we employ the standard artificial viscosity [24],

$$
\Pi_{i j}=\left\{\begin{array}{ll}
\frac{-\alpha \overline{c_{i j}} \phi_{i j}+\beta \phi_{i j}^{2}}{\overline{\rho_{i j}}}, & \boldsymbol{v}_{i j} \cdot \boldsymbol{x}_{i j}<0 \\
0, & \boldsymbol{v}_{i j} \cdot \boldsymbol{x}_{i j} \geqslant 0
\end{array},\right.
$$

where

$$
\phi_{i j}=\frac{h_{i j} v_{i j} x_{i j}}{\left|x_{i j}\right|^{2}+\varphi^{2}},
$$

$$
\overline{c_{i j}}=\frac{1}{2}\left(c_{i}+c_{j}\right),
$$

$\bar{\rho}_{i j}=\frac{1}{2}\left(\rho_{i}+\rho_{j}\right)$,

$h_{i j}=\frac{1}{2}\left(h_{i}+h_{j}\right)$,

$\boldsymbol{v}_{i j}=\boldsymbol{v}_{i}-\boldsymbol{v}_{j}, \quad \boldsymbol{x}_{i j}=\boldsymbol{x}_{i}-\boldsymbol{x}_{j}$.

In the above equations, $\alpha$ and $\beta$ are constants that are all typically set around 1.0. The factor $\varphi=0.1 h_{i j}$ is inserted to prevent numerical divergences between two particles are approaching each other. $c$ and $\boldsymbol{v}$ represent the speed of sound and the particle velocity vector, respectively. The viscosity term associated with $\alpha$ produces a bulk viscosity, while the second term associated with $\beta$, which is intended to suppress particle interpenetration at high Mach number, is similar to the von Neumann-Richtmyer artificial viscosity.

\subsection{Kernel gradient correction}

It is known that the conventional SPH method has been hindered with low accuracy as it cannot exactly reproduce quadratic and linear functions, and even cannot exactly reproduce a constant. The accuracy of the conventional SPH method is also closely related to the distribution of particles, selection of smoothing function and the support domain. During the last decade, different approaches have been proposed to improve the particle inconsistency and hence the SPH approximation accuracy. Some of them involve reconstruction of a new smoothing function so as to satisfy the discretized consistency conditions. However, these approaches are usually not preferred for hydrodynamic simulations because the reconstructed smoothing function can be partially negative, non-symmetric, and not monotonically decreasing. Recently, one popular way is to construct improved SPH approximation schemes based on Taylor series expansion on the SPH approximation of a function and/or its derivatives.

The process of shaped charge explosion, metal jet formation and penetration involve fast expansion of explosive gas, rapid deformation and even liquefaction of metal case and liner, and quick damage on target materials. These lead to highly disordered particle distribution, which can seriously influence computational accuracy of SPH approximations. Hence an SPH approximation scheme, which is of higher order accuracy and is insensitive to disordered particle distribution, is necessary for modeling shaped charges. In this paper, the kernel gradient in SPH approximations is improved with a kernel gradient correction (KGC) technique [34]. In the KGC technique, a modified or corrected kernel gradient is obtained by multiplying the original kernel gradient with a local reversible matrix $L\left(\mathbf{r}_{i}\right)$, which is obtained from Taylor series expansion method. In two-dimensional spaces, the new kernel gradient of the smoothing function $\nabla_{i}^{C} W_{i j}$ can be obtained as follows

$\nabla_{i}^{C} W_{i j}=L\left(\mathbf{r}_{i}\right) \nabla_{i} W_{i j}$

$$
L\left(\mathbf{r}_{i}\right)=\left(\sum_{j}\left(\begin{array}{cc}
x_{j i} \frac{\partial W_{i j}}{\partial x_{i}} & y_{j i} \frac{\partial W_{i j}}{\partial x_{i}} \\
x_{j i} \frac{\partial W_{i j}}{\partial y_{i}} & y_{j i} \frac{\partial W_{i j}}{\partial y_{i}}
\end{array}\right) V_{j}\right)^{-1},
$$

where $x_{j i}=x_{j}-x_{i}, y_{j i}=y_{j}-y_{i}$, where $x, y$ are the two components of the position vector $\boldsymbol{x}$. It is found that for general cases with irregular particle distribution, variable smoothing length, and/or truncated boundary areas, the SPH particle approximation scheme with kernel gradient correction is of second order accuracy. It is noted that for gradient correction, since only the kernel gradient are corrected, there is no need to significantly change the structure of SPH computer programs and procedure of SPH simulations. It is therefore convenient in implementing the SPH equations of motion.

\subsection{Treatment of multi-material}

When using the SPH method to model the entire process of HE detonation and explosion, explosion-driven metal deformation and jet formation as well the penetrating effects, a major challenge in numerical simulation is that the treatment of multi-materials (explosive gas, metal case and liner) with large density ratios, which can introduce large numerical oscillations in the interface areas. As such when modeling problems with multi-materials, it is necessary to render the interface conditions to ensure the pressure and normal velocity on both sides of the interface to be continuous. In SPH simulation, particles from different materials can interact with each other, and when approximating field variables of a particle from one material, neighboring particles from different material can contribute in the approximation process (as shown in Fig. 4). According to Eqs. (1) and (2), the interaction of neighboring interface particles implicitly implements the interface conditions (equal pressure and normal velocity). With the averaging effects of the SPH approximation, field properties such as pressure and normal velocity tend to roughly equal (not exactly as in the gridbased methods). This implicitly implemented interface treatment is dependent on the accuracy of the SPH method. For conventional SPH method, as the computational accuracy is poor (especially for disordered particles), there may be large discrepancies between the field variables on both sides of the material interface. For the presented SPH approximation with kernel gradient correction, as the computational accuracy is comparatively higher, the obtained approximation results for field variables can be very close on both sides of the material interface. This is the major reason that using conventional SPH is usually not able to obtain reasonable results when modeling shaped charge with multi-material (high explosive, metal case and liner), while the modified SPH model with KGC makes this mission possible.

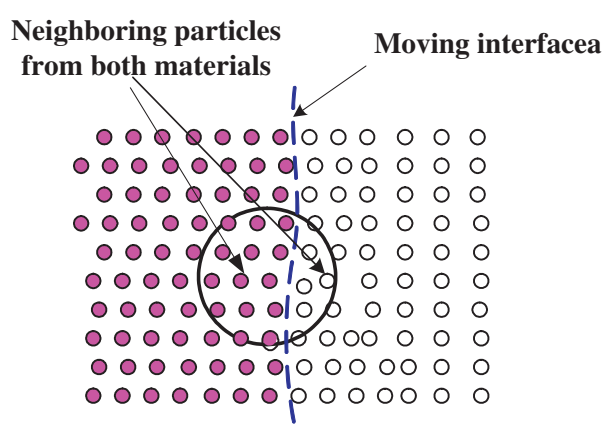

Material I

Material II

Fig. 4. Treatment of multi-material with moving material interfaces. 


\section{Constitutive modeling and equation of state}

\subsection{Constitutive modeling}

Johnson-Cook model [35] is one of the most popular constitutive models for numerical simulations of impact and penetration, and which are usually associated with high strain rate. The model considers the effects of the stress hardening, strain rate and the temperature evolution. The yield stress in Johnson-Cook model can be written as

$\sigma_{y}=\left(A+B \varepsilon^{p^{n}}\right)\left(1+C \ln \dot{\varepsilon}^{*}\right)\left(1-T^{* m}\right)$,

$T^{*}=\frac{T-T_{\text {room }}}{T_{\text {melt }}-T_{\text {room }}}$,

where $\varepsilon^{p}$ is the effective plastic strain, $\dot{\varepsilon}^{*}$ is a dimensionless strain rate, and $T$ is the temperature. $A, B, C, n$ and $m$ are five parameters in the Johnson-Cook model that need to be determined from the torsion test under different strain rate, the Hopkinson Pressure Bar test with different temperatures, and the Standard static tensile test. Detail parameters in the Johnson-Cook model used in this paper are listed in Tables 1 and 2 for aluminum and the steel respectively.

\subsection{Equation of state (EOS)}

For explosive gas, the standard Jones-Wilkins-Lee (JWL) equation [36] of state can be employed. The pressure of the explosive gas is given by

$p=A\left(1-\frac{\omega \eta}{R_{1}}\right) e^{-\frac{R_{1}}{\eta}}+B\left(1-\frac{\omega \eta}{R_{2}}\right) e^{-\frac{R_{2}}{\eta}}+\omega \eta \rho_{0} E$,

where $\eta$ is the ratio of the density of the explosive gas to the initial density of the original explosive. $e$ is the internal energy of the high explosive per unit mass. $A, B, R_{1}, R_{2}$ and $\omega$ are coefficients obtained by fitting the experimental data. $E$ is the initial internal energy of the high explosive per unit mass. Values of the corresponding coefficients are listed in Table 3.

The Tillotson equation [37] is employed to describe pressurevolume-energy behavior of metals under high temperature, pressure and strain rate. The coordinate plane of the pressure-specific volume is divided into four regions which represent four different phases of the material, e.g., the solid phase area, the liquid phase area, the vapor and liquid mixture area, and the vapor phase area in the Tillotson equation (illustrated in Fig. 5).

As such, the Tillotson equation describes a wide range of the dynamic behavior of material even with a complicated phase transition. The pressures of the four phases are given as

$$
\begin{aligned}
& p_{1}=\left(a+\frac{b}{\omega_{0}}\right) \eta \rho_{0} e+A \mu+B \mu^{2} \\
& p_{2}=\left(a+\frac{b}{\omega_{0}}\right) \eta \rho_{0} e+A \mu \\
& p_{3}=p_{2}+\frac{\left(p_{4}-p_{2}\right)\left(e-e_{s}\right)}{\left(e_{s}^{\prime}-e_{s}\right)} \\
& p_{4}=a \eta \rho_{0} e+\left(\frac{b \eta \rho_{0} e}{\omega_{0}}+A \mu e^{\beta x}\right) e^{-\alpha x^{2}} \\
& \eta=\frac{\rho}{\rho_{0}}, \quad \mu=\eta-1, \quad \omega_{0}=1+\frac{e}{e_{0} \eta^{2}}
\end{aligned}
$$

where $a, b, A, B, \alpha, \beta, e_{0}, e_{s}$ and $e^{\prime}$ are the parameters determined by the material, and $p_{1}$ to $p_{4}$ are the pressure of the above-mentioned

Table 1

Parameters used in the Johnson-Cook model for aluminum.

\begin{tabular}{lllllll}
\hline$A(\mathrm{MPa})$ & $B(\mathrm{MPa})$ & $C$ & $n$ & $m$ & $T_{\text {room }}(\mathrm{K})$ & $T_{\text {melt }}(\mathrm{K})$ \\
\hline 175 & 380 & 0.0015 & 0.34 & 1.0 & 273 & 775 \\
\hline
\end{tabular}

Table 2

Parameters used in the Johnson-Cook model for steel.

\begin{tabular}{lllllll}
\hline$A(\mathrm{MPa})$ & $B(\mathrm{MPa})$ & $C$ & $n$ & $m$ & $T_{\text {room }}(\mathrm{K})$ & $T_{\text {melt }}(\mathrm{K})$ \\
\hline 350 & 275 & 0.022 & 0.36 & 1.0 & 273 & 1573 \\
\hline
\end{tabular}

Table 3

Parameters used in the JWL equation for TNT

\begin{tabular}{lllllll}
\hline$\rho_{0}\left(\mathrm{~kg} \mathrm{~m}^{-3}\right)$ & $A(\mathrm{GPa})$ & $B(\mathrm{GPa})$ & $R_{1}$ & $R_{1}$ & $\omega$ & $E\left(\mathrm{~kJ} \mathrm{~kg}^{-1}\right)$ \\
\hline 1630 & 317.2 & 3.21 & 4.15 & 0.95 & 0.30 & 4290 \\
\hline
\end{tabular}

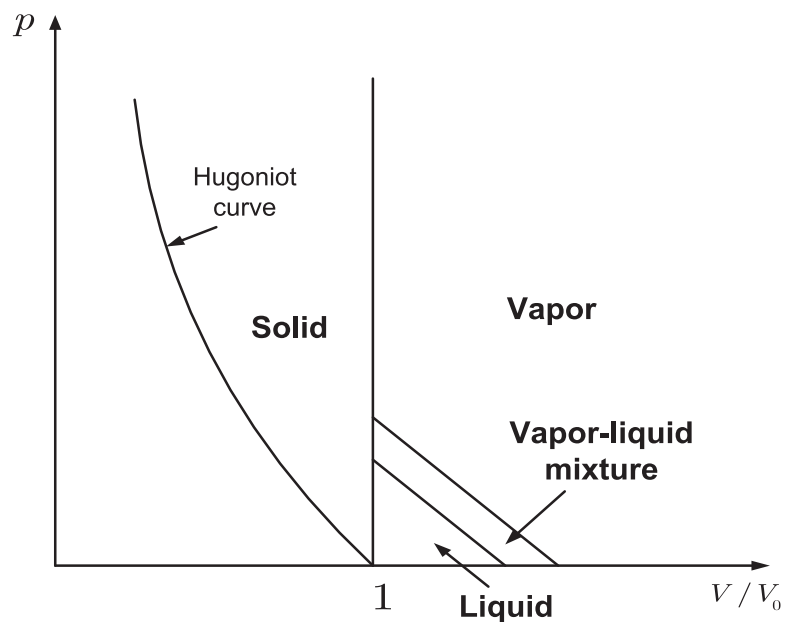

Fig. 5. Pressure-special volume plane of the Tillotson equation.

four phases. Listed here in Tables 4 and 5 are the parameters of aluminum and steel for the Tillotson equation.

\section{Numerical examples}

In this section, the modified SPH method and an in-house SPH code shall be used to model three numerical examples, the detonation process of a TNT slab, jet formation of a linear shaped charge and the penetrating and damaging effects on a steel target plate induced by a linear shaped charge jet. The obtained SPH results shall be compared with experimental observations.

\subsection{One-dimensional TNT slab detonation}

In this example, a $0.1 \mathrm{~m}$ long TNT slab is detonated at one end with a constant detonation velocity of $6930 \mathrm{~m} / \mathrm{s}$ (illustrated in Fig. 6). In the simulation, the symmetric condition is used. Therefore the detonation of the $0.1 \mathrm{~m}$ long slab from one end to the other is equivalent to the detonation of a $0.2 \mathrm{~m}$ long slab that is ignited at the middle point and advances to both ends [26]. Before the detonation, particles are evenly distributed along the slab. There are 4000 particles used in this simulation and the initial smoothing length is taken as 1.2 times of the initial particle distance. The smoothing length of each particle is updated in the formulation presents by Benz [38], and the parameters $\alpha$ and $\beta$ used in the artificial viscosity are taken as 1.0 and 10 respectively.

According the detonation velocity, it takes about $14.4 \mu \mathrm{s}$ to complete the detonation throughout the TNT slab. Fig. 7 shows the pressure profiles along the slab at $1 \mu$ s interval from 1 to $14 \mu \mathrm{s}$. It is seen that with the process of the detonation, the detonation pressure converges to the experimentally determined C-J pressure, which is, according to Chapman and Jouguet's 
Table 4

Parameters used in the Tillotson equation for aluminum.

\begin{tabular}{|c|c|c|c|c|c|c|c|c|}
\hline$A(\mathrm{GPa})$ & $B(\mathrm{GPa})$ & $a$ & $b$ & $\alpha$ & $\beta$ & $e_{0}\left(\mathrm{~kJ} \mathrm{~g}^{-1}\right)$ & $e_{s}\left(\mathrm{~kJ} \mathrm{~g}^{-1}\right)$ & $e^{\prime}\left(\mathrm{kJ} \mathrm{g}^{-1}\right)$ \\
\hline 75.20 & 65.00 & 0.50 & 1.63 & 5.00 & 5.00 & 5.00 & 3.00 & 15.00 \\
\hline
\end{tabular}

Table 5

Parameters used in the Tillotson equation for steel.

\begin{tabular}{|c|c|c|c|c|c|c|c|c|}
\hline$A(\mathrm{GPa})$ & $B(\mathrm{GPa})$ & $a$ & $b$ & $\alpha$ & $\beta$ & $e_{0}\left(\mathrm{~kJ} \mathrm{~g}^{-1}\right)$ & $e_{s}\left(\mathrm{~kJ} \mathrm{~g}^{-1}\right)$ & $e^{\prime}\left(\mathrm{kJ} \mathrm{g}^{-1}\right)$ \\
\hline 127.90 & 105.00 & 0.50 & 1.63 & 5.00 & 5.00 & 9.50 & 2.44 & 10.20 \\
\hline
\end{tabular}

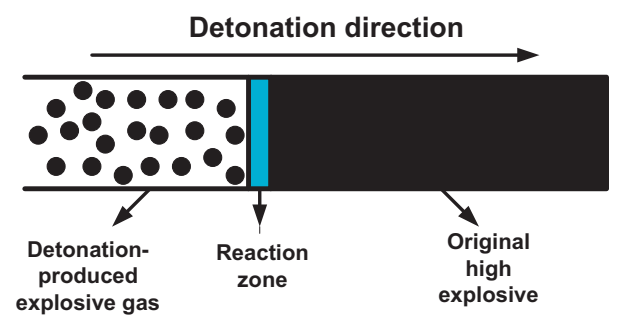

Fig. 6. The detonation of a 1D TNT slab.

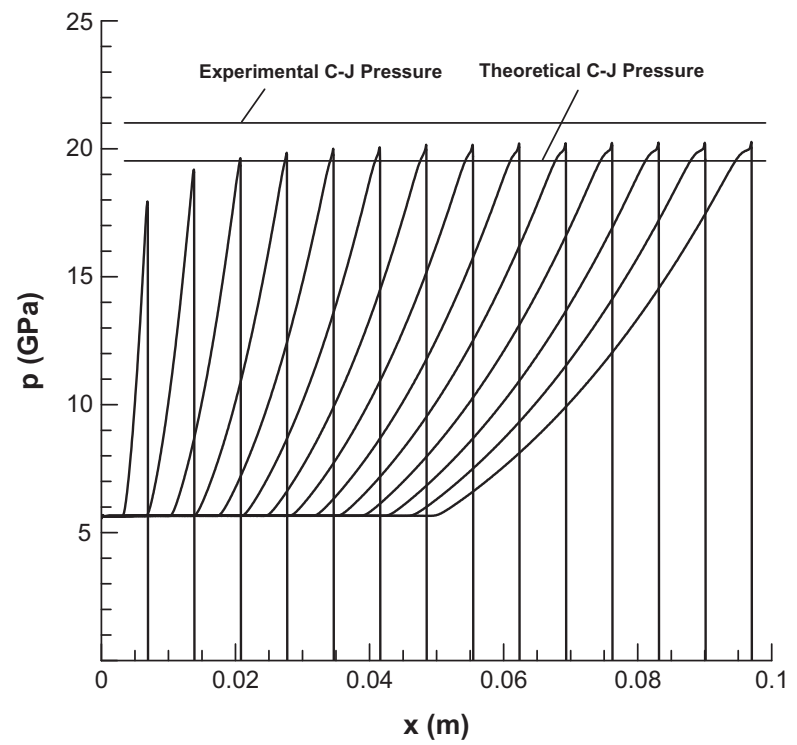

Fig. 7. Pressure profiles along the 1-D TNT slab during the detonation process.

hypothesis, the pressure at the tangential point of the Hugoniot curve and the Rayleigh line, and represents the pressure at the equilibrium plane at the trailing edge of the very thin chemical reaction zone. The peak shock pressure is also close to theoretical C-J pressure [17] with slightly bigger values.

\subsection{Metal jet formation of a linear shaped charge}

After the detonation of a shaped charge, the detonation produced high pressure explosive gas produces tremendous loadings on the surrounding metal case and liner, which will lead to large deformation and even liquefaction of the material and form an evolutionary metal jet. In this example, the detonation and explosion of high explosive charge, the interaction of explosive gas and surrounding metal structures, and the formation of metal jet shall be simulated using the modified SPH method. The simulation involves multi-material (explosive-metal) and multi-phase (solid-gas-liquid) with moving features, large deformation and strong fluid-structure interaction, and is a formidable task for conventional grid-based numerical methods.

Early investigations on shaped charges seldom consider the effect of the metal case, since it is commonly believed that the formation of the metal jet is mainly related to the pressure generated by the explosive gas around the liner, and the effects of metal case on the metal jet is negligible. Some shaped charge devices even do not have a case at the stage of experimental design, or only a very thin shell is placed as a case to contain the explosive, and the strength of the thin shell can be ignored compared with the pressure of the explosive. As an important structure in a practical shaped charge device, how does the metal case influence the formation of metal jet and how much is its influence? These still remain as unresolved problems. In this section, we shall provide an in-depth study to identity these problems by comparatively investigating the shaped charge metal jet formation with and without metal case.

Firstly, a conventional computational model without metal case is applied to simulate the formulation of the shaped charge jet using the modified SPH method with kernel gradient correction. In this example, the geometry model is a shaped charge with an

Table 6

Jet velocities vs particle numbers.

\begin{tabular}{lllll}
\hline $\begin{array}{l}\text { Total } \\
\text { number of } \\
\text { particles }\end{array}$ & $\begin{array}{l}\text { Numbers of } \\
\text { particles for TNT } \\
\text { explosive }\end{array}$ & $\begin{array}{l}\text { Numbers of } \\
\text { particles for } \\
\text { liner }\end{array}$ & $\begin{array}{l}\text { Normalized } \\
\text { velocity }\end{array}$ & $\begin{array}{l}\text { Error } \\
(\%)\end{array}$ \\
\hline 3424 & 2624 & 800 & 0.9928 & 0.72 \\
4116 & 3156 & 960 & 0.9971 & 0.29 \\
5176 & 3784 & 1392 & 1.0000 & 0 \\
6158 & 4744 & 1414 & 1.0004 & 0.04 \\
7716 & 5916 & 1800 & 1.0014 & 0.14 \\
\hline
\end{tabular}

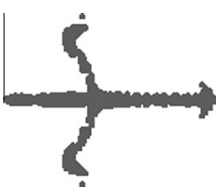

Fig. 8. Shape of aluminum jet at $30 \mu$ s (Present SPH results).

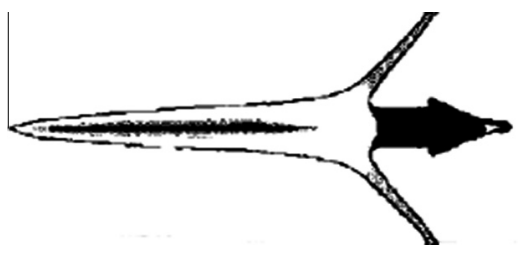

Fig. 9. Shape of aluminum jet (from Ref. [37]). 


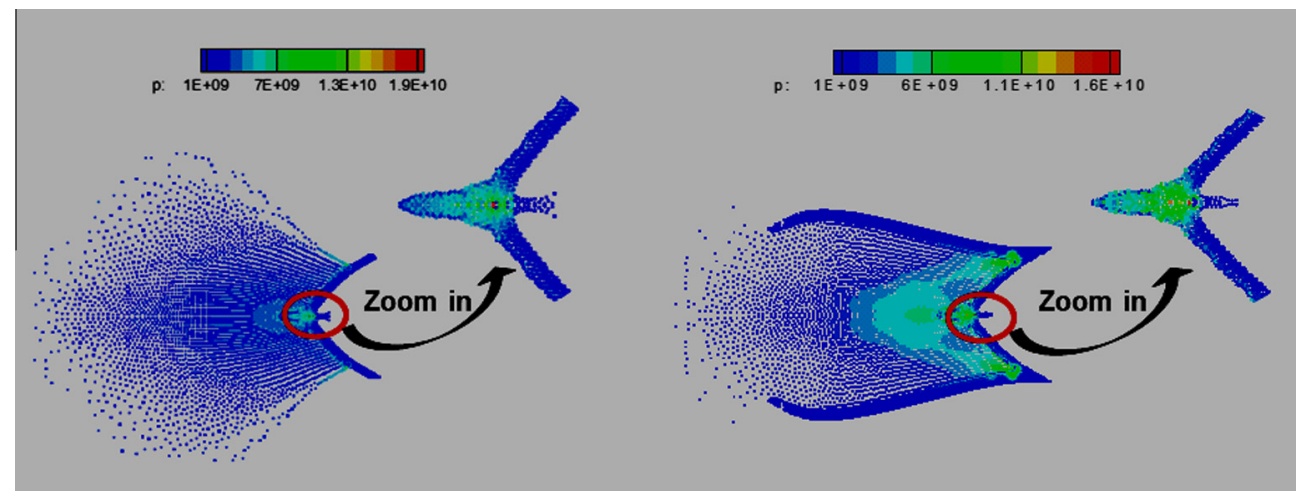

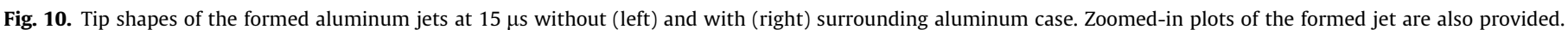

aluminum liner of $3 \mathrm{~mm}$-thick, and a cone angel of $60^{\circ}$. The explosive is $48 \mathrm{~mm}$ in width and $100 \mathrm{~mm}$ in length. 3784 Particles are used to model the TNT explosive and 1392 particles are used to model the metal liner. The jet velocities obtained from different particles resolutions are shown in Table 6 . The velocity magnitudes are normalized by the jet velocity of $5148 \mathrm{~m} / \mathrm{s}$ with 5176 particles (3784 particles for TNT explosive and 1392 particles for liner). It is demonstrated that adequate number of particles have been used to obtain a converged result.

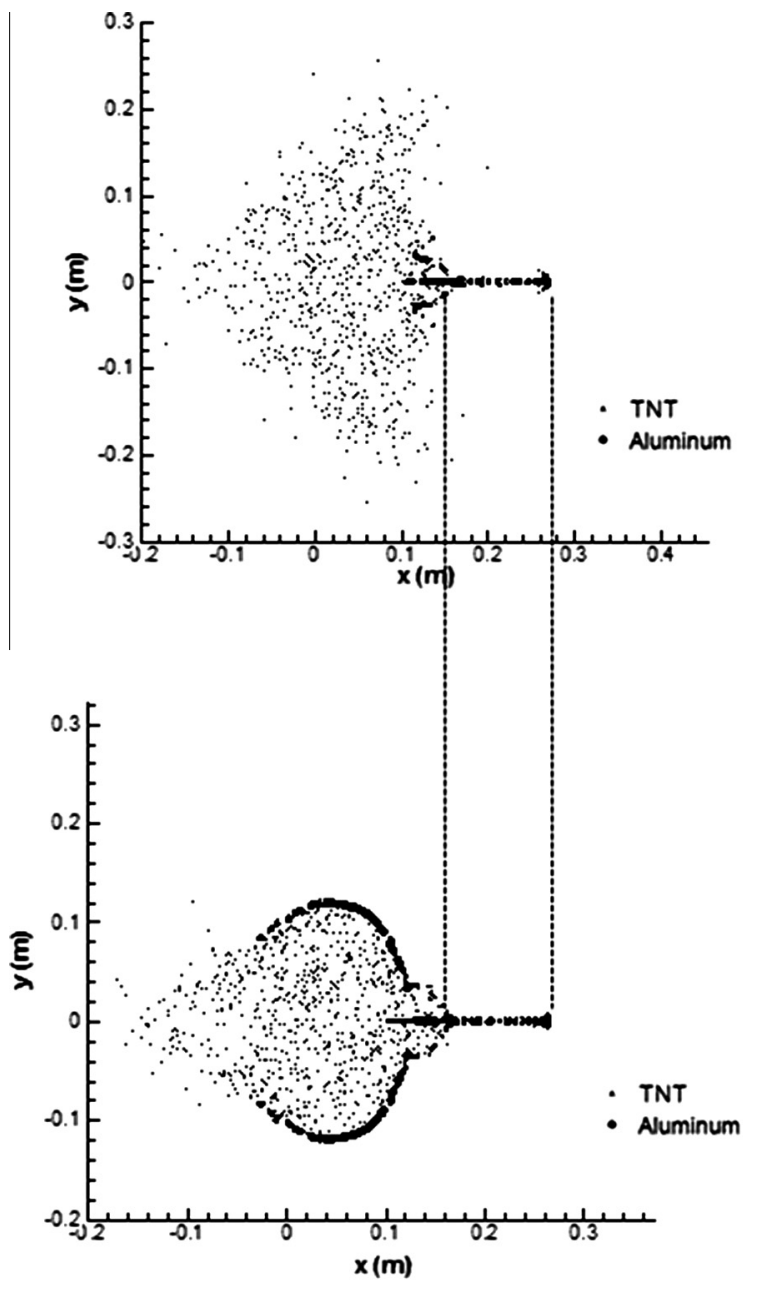

Fig. 8 shows the shape of the formed aluminum jet (explosive gas particles are not shown), which is close to that obtained from experimental and computational observation by Katayama et al. (illustrated in Fig. 9) [37].

Secondly, a shaped charge model with a $5 \mathrm{~mm}$-thick aluminum case is numerically simulated using the same SPH model and geometry parameters as previous one. 4174 more particles are employed in order to simulate the aluminum case. Fig. 10 shows the comparisons of tip shapes of the formed aluminum jets at $15 \mu \mathrm{s}$

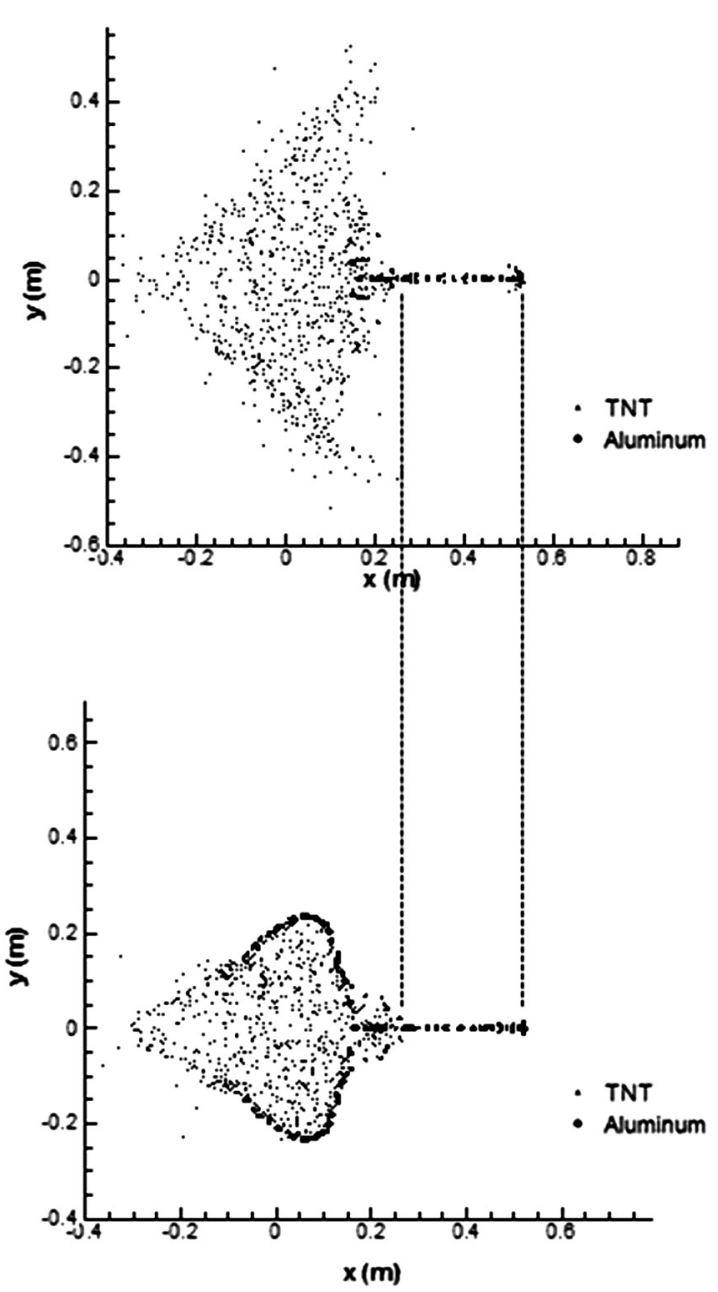

Fig. 11. Comparison of the two computational models at $50 \mu \mathrm{s}$ and $100 \mu \mathrm{s}$. 

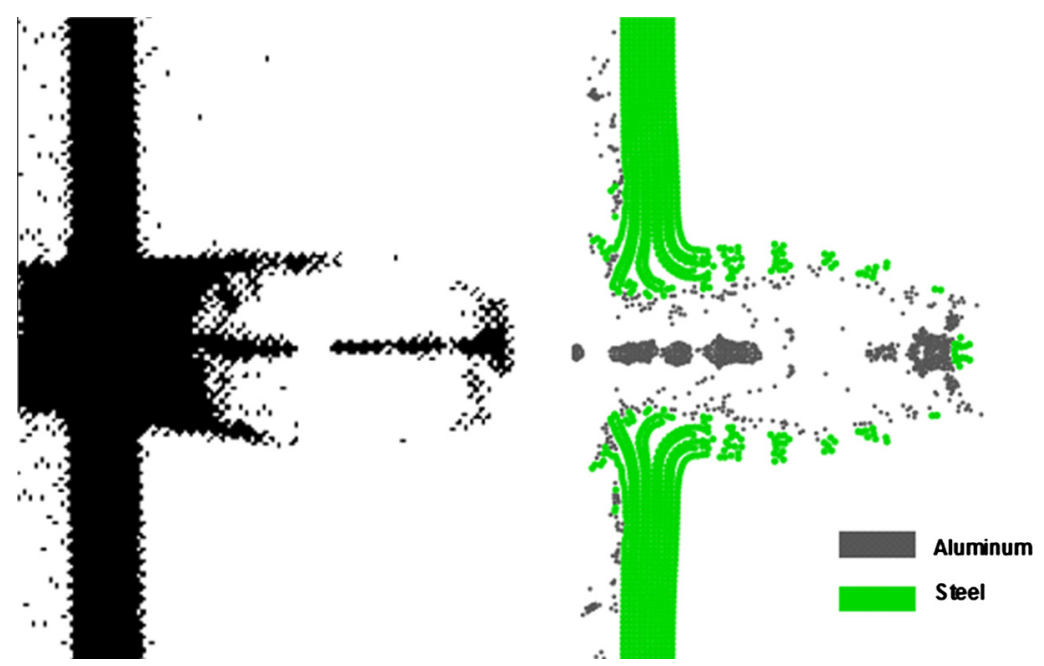

Fig. 12. Comparison of the experimental radiophoto (left) with the SPH simulation results (right) of the penetration process.

without (left) and with (right) surrounding aluminum case. It is seen that with surrounding aluminum case, the obtained jet tip is sharper than that obtained without surrounding aluminum case. As the surrounding aluminum case acts to confine the expansion of the explosive gas, and thus shaped charge with surrounding cases can leads to stronger converging effects with sharper jet tip. If there is no surrounding case, rarefaction wave comes from outside, and leads to smaller pressure behind the detonation wave. Therefore, shaped charge without surrounding cases may produce obtuse jet tip.

Fig. 11 shows a more detailed comparison of the two computational models on the evolution of shaped charge particles and characteristics of the formed jet (shape, length and velocity) at $50 \mu \mathrm{s}$ and $100 \mu$ s respectively. For these two different cases, the obtained snapshots of explosive gas expansion are different. If confined in metal case, the expansion of the explosive gas is seriously restricted and gas particles moves faster at the end without cavity. Though the obtained shapes of jet tips are slightly different for these two models, the obtained jet lengths are nearly the same, and the obtained velocities at the jet tips are also very close (Approximately $5.2 \mathrm{~km} / \mathrm{s}$ at both $50 \mu \mathrm{s}$ and $100 \mu \mathrm{s}$ ).

\subsection{Damaging and penetrating effects due to a jet from a linear shaped charge}

In this section, a metal jet produced from the detonation and explosion of a linear shaped charge is used to penetrate a steel plate. The shaped charge is deployed with an aluminum case. The computational geometry, particle setup and parameters for the shaped charge are same as in the previous example. The steel plate is as $100 \mathrm{~mm}$ in length and $10 \mathrm{~mm}$ in width. The whole simulation uses 11,350 particles in total.

Fig. 12 shows the distribution of shaped charge particles and debris cloud as the aluminum jet penetrating onto the steel plate at $70 \mu \mathrm{s}$. The pinnate debris cloud during the penetration process and the local gathering of the jet tip after penetration can be clearly observed. In the zoomed-in plot in the penetrating region, the size and shape of the hole and debris cloud obtained from SPH simulation agree well with experimental observations by Raftenberg, who studied the process of a shaped charge warhead, made by OFHC copper, fired into an RHA (Rolled Homogeneous Armor) plate with a series of radiographs [6].

Fig. 13 shows the time history of energy evolution during the aluminum jet formation and penetration onto the steel plate. It is

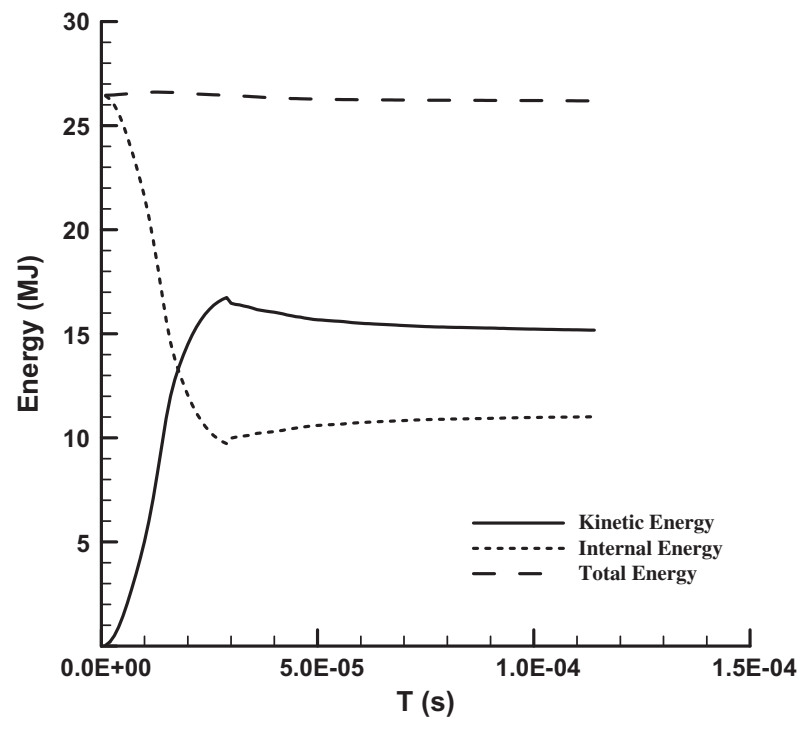

Fig. 13. Energy evolution during the penetration process.

observed that during the SPH simulation, the kinetic energy of the system increases rapidly and the internal energy decreases before $24 \mu \mathrm{s}$. The aluminum jet is produced during this period of time, and the internal energy of the HE is converted into kinetic energy. At around $24 \mu \mathrm{s}$, both the internal energy and the kinetic energy of the system are associated with a quick jump, which corresponds to the sudden penetration of the aluminum jet onto the steel plate. During the penetration process, the kinetic energy decreases slightly and the internal energy increases slightly. After completing the penetration process, both the kinetic energy and internal energy keep nearly constant. During the entire process, the total energy is nearly constant (within an error of $1 \%$ ). This shows a good conservation of energy and can serve as a validation of the SPH model.

\section{Summary and conclusion}

This paper presents a numerical simulation of linear shaped charge using a smoothed particle hydrodynamics (SPH) method, in which a modified scheme for approximating kernel gradient (kernel gradient correction, or KGC) is implemented. The entire 
process of HE detonation and explosion, explosion-driven metal deformation and jet formation as well the penetrating effects is modeled and compared with experimental observations. It is demonstrated that

(1) The modified SPH method can effectively treat multi-materials and multi-phases with moving features, large deformation and strong fluid-structure interactions.

(2) For computational models with and without metal case, the obtained jet length and velocity are nearly the same, though the obtained shapes of the jet tip can be different.

(3) During the entire process of HE detonation and explosion, explosion-driven metal deformation and jet formation and penetration, the total energy of the system basically keeps constants, which also shows the reliability of the SPH model.

\section{Acknowledgements}

This work has been supported by the National Natural Science Foundation of China (11172306) and National Defense Innovation Funds of the Chinese Academy of Sciences (Y175031XML).

\section{References}

[1] Walters WP, Zukas JA. Fundamentals of shaped charges. Wiley; 1989.

[2] Birkhoff G, MacDougall DP, Pugh EM, Taylor SG. Explosives with lined cavities. J Appl Phys 1948;19:563-82.

[3] Pugh EM, Eichelberger RJ, Rostoker N. Theory of jet formation by charges with lined conical cavities. J Appl Phys 1952;23:532-6.

[4] Eichelberger RJ. Experimental test of the theory of penetration by metallic jets. J Appl Phys 1956;27:63-8.

[5] Green RE. First X-ray diffraction photograph of a shaped charge jet. Rev Sci Instrum 1975;46:1257-61.

[6] Raftenberg MN. Experimental investigation of RHA plate perforation by a shaped-charge jet. In: Chou SC, editor. Twelfth army symposium on solid mechanics proceedings. Plymouth, Massachusetts, USA; 1992. p. 395-410.

[7] Yu C, Tong YJ, Yan CL, Li FB, Gui YL, Zhang M, et al. Applied research of shaped charge technology. Int J Impact Eng 1999;23:981-8.

[8] Wang C, Fu XL, Ning JG. Numerical simulation of shaped charge jet formation under different ways of initiation. Trans Beijing Inst Technol 2006;26:401-4.

[9] Katayama M, Kibe S, Yamamoto T. Numerical and experimental study on the shaped charge for space debris assessment. Acta Astronaut 2001:48:363-72.

[10] Birnbaum N, Cowler M, Itoh M, Katayama M, Obata H. AUTODYN - an interactive non-linear dynamic analysis program for microcomputer through supercomputers. In: Ninth international conference on structural mechanics in reactor technology. Lausanne Switzerland; 1987. p. 401-6.

[11] Chou PC, Ciccarelli RD, Walters WP. The formation of jets from hemisphericalliner warheads. In: 7th International symposium on ballistics. The Hague, Netherlands; 1983.

[12] Hallquist JQ. DYNA3D user's manual (nonlinear dynamic analysis of solids in three dimensions). Lawrence Livermore National Laboratory; 1986.

[13] Johnson GR. Recent developments and analyses associated with the EPIC-2 and EPIC-3 codes. In: Wang SS, Renton WJ, editors. Advances in aerospace structures and materials, AD-01. New York: ASME; 1981.
[14] Hageman LJ, Walsh JM. HELP, a multi-material Eulerian program for compressible fluid and elastic-plastic flows in two space dimensions and time. System, Science and Software Inc.; 1975.

[15] MSC/DYTRAN user's manual, 4 ed. USA: The MacNeal-Schwndler Corporation; 1997.

[16] Ma S, Zhang X, Lian Y, Zhou X. Simulation of high explosive explosion using adaptive material point method. Comput Model Eng Sci (CMES) 2009;39:101.

[17] Shin YS, Chisum JE. Modelling and simulation of underwater shock problems using a coupled Lagrangian-Eulerian analysis approach. Shock Vib 1997;4:1-10

[18] Liu GR, Lam KY, Lu C. Computational simulation of sympathetic explosion in a high performance magazine. In: Proceeding of third weapon effects seminar. Singapore; 1998. p. 65-75.

[19] Molinari JF. Finite element simulation of shaped charges. Finite Elem Anal Des 2002;38:921-36

[20] Ayisit O. The influence of asymmetries in shaped charge performance. Int J Impact Eng 2008;35:1399-404.

[21] Karlsson HEV. Computer simulation of shaped charge jet fragmentation. In: 20th International symposium on ballistics. Orlando, FL, USA; 2002. p. 557-64.

[22] Lucy LB. A numerical approach to the testing of the fission hypothesis. Astron J 1977;82:1013-24.

[23] Gingold RA, Monaghan JJ. Smoothed particle hydrodynamics-theory and application to non-spherical stars. Mon Not R Astron Soc 1977;181:375-89.

[24] Liu GR, Liu MB. Smoothed particle hydrodynamics: a meshfree particle method. Singapore: World Scientific; 2003.

[25] Liu MB, Liu GR. Smoothed particle hydrodynamics (SPH): an overview and recent developments. Arch Comput Method Eng 2010;17:25-76.

[26] Liu MB, Liu GR, Zong Z, Lam KY. Computer simulation of high explosive explosion using smoothed particle hydrodynamics methodology. Comput Fluids 2003;32:305-22.

[27] Liu MB, Liu GR, Lam KY, Zong Z. Smoothed particle hydrodynamics for numerical simulation of underwater explosion. Comput Mech 2003;30:106-18.

[28] Swegle JW, Attaway SW. On the feasibility of using smoothed particle hydrodynamics for underwater explosion calculations. Comput Mech 1995; 17:151-68.

[29] Randles PW, Libersky LD. Smoothed particle hydrodynamics: some recent improvements and applications. Comput Meth Appl Mech Eng 1996;139:375-408.

[30] Liu MB, Liu GR, Lam KY. Adaptive smoothed particle hydrodynamics for high strain hydrodynamics with material strength. Shock Waves 2006;15:21-9.

[31] Monaghan JJ. Smoothed particle hydrodynamics. Annu Rev Astron Astrophy 1992;30:543-74.

[32] Liu MB, Liu GR, Lam KY, Zong Z. Meshfree particle simulation of the detonation process for high explosives in shaped charge unlined cavity configurations. Shock Waves 2003;12:509-20.

[33] Yang G, Han X, Hu DA. Computer simulation of two-dimensional linear-shaped charge jet using smoothed particle hydrodynamics. Eng Comput 2011;28:58-75.

[34] Shao JR, Li HQ Liu GR, Liu MB. An improved SPH method for modeling liquid sloshing dynamics. Comput Struct 2012;100-101:18-26.

[35] Johnson GR, Cook WH. A constitutive model and data for metals subjected to large strains, high strain rates and high temperatures. In: Proceedings of seventh international symposium on ballistics. The Hague, Netherlands: International Ballistics Committee; 1983. p. 541-7.

[36] Lee EL, Hornig HC, Kury JW. Adiabatic expansion of high explosive detonation products. California Univ., Livermore. Lawrence Radiation Lab.; 1968.

[37] Katayama M, Takeba A, Toda S, Kibe S. Analysis of jet formation and penetration by conical shaped charge with the inhibitor. Int J Impact Eng 1999;23:443-54.

[38] Benz W. Smooth particle hydrodynamics - a review. Numerical modeling of non-linear stellar pulsation: problems and prospects. Boston: Kluwer Academic; 1990. p. 269. 